\title{
Thermal Flow Sensor for Non-Invasive Measurements in HVAC Systems ${ }^{\dagger}$
}

\author{
Samir Cerimovic 1,*, Albert Treytl ${ }^{1}$, Thomas Glatzl ${ }^{1}$, Roman Beigelbeck ${ }^{1}$, Franz Keplinger ${ }^{2}$ and \\ Thilo Sauter ${ }^{1}$ \\ 1 Department for Integrated Sensor Systems, Danube University Krems, A-2700 Wiener Neustadt, Austria; \\ albert.treytl@donau-uni.ac.at (A.T.); thomas.glatzl@donau-uni.ac.at (T.G.); \\ roman.beigelbeck@donau-uni.ac.at (R.B.); thilo.sauter@donau-uni.ac.at (T.S.) \\ 2 Institute of Sensor and Actuator Systems, Vienna University of Technology, A-1040 Vienna, Austria; \\ franz.keplinger@tuwien.ac.at \\ * Correspondence: samir.cerimovic@donau-uni.ac.at; Tel.: +43-2622-2342014 \\ † Presented at the Eurosensors 2018 Conference, Graz, Austria, 9-12 September 2018.
}

Published: 24 December 2018

\begin{abstract}
We present a feasibility study on non-invasive flow rate measurements in heating, ventilation, and air conditioning (HVAC) systems utilizing thermal transduction instead of commonly used ultrasonic techniques. The investigated thermal flow transduction comprises two temperature sensors and a heater, all mounted non-invasively on the outer pipe surface and, therefore, not disturbing the fluid flow inside. One temperature sensor measures the heater temperature, whereas the other one, mounted upstream of the heater, follows the fluid temperature for reference. The temperature difference (i.e., the heater excess temperature) depends on the fluid velocity and can be used to derive the mean volume flow inside the pipe. In order to visualize and study the temperature field, a finite element method (FEM) model of the system was implemented. Measurements conducted with the sensor prototype confirm the feasibility of this approach.
\end{abstract}

Keywords: thermal flow sensor; non-invasive flow rate measurement; HVAC systems

\section{Introduction}

Systems for heating, ventilation, and air conditioning (HVAC) contribute significantly to the overall energy consumption of modern buildings. Some analyses show that up to $40 \%$ of energy could be saved by improved control strategies of such systems [1]. In order to optimize the energy consumption of a building or to detect atypical behaviors, information on the energy flow within the HVAC system is required. For this purpose, fluid temperature and flow velocity must be obtained at distinctive points in the hydraulic circuit. These data are usually collected using invasive temperature and flow sensors. However, modifications of an existing hydraulic system are often not desirable or possible (e.g., due to legal matters). In this case, non-invasive clamp-on temperature sensors as well as ultrasonic (US) flow sensors can be used. Besides their high price, US flow sensors are less suitable for smaller pipe diameters. Searching for a promising alternative, we have studied the utilization of low-cost thermal flow sensors to hydraulic circuits with metalpipes.

According to the underlying physical principle, there are three different types of thermal flow sensors $[2,3]$ :

1. Hot-wire or hot-film flow sensors, which exploit directly the cooling effect of the passing fluid on a heater. Here, the heater excess temperature (i.e., the difference between heater and fluid temperature) corresponds to the flow velocity.

2. Calorimetric flow sensors utilize the flow dependent asymmetry of the temperature profile 
around the heater. In this case, temperature sensors arranged around the heater are needed for a detection. Their temperature difference is a function of the flow velocity.

3. Time-of-flight (TOF) flow sensors measure the propagation of a heat pulse over a known distance between the heater and the temperature sensor located downstream of the heater.

Due to high thermal conductivity of metal pipes, the excess temperature along the pipe surface drops significantly with increasing distance from the heater, even if very high heating power is applied. Hence, calorimetric and TOF sensors are less suitable for non-invasive measurements on metal pipes. In the course of this feasibility study, we therefore concentrated on hot wire transduction and developed a prototype, which was tested on common copper pipes. In contrast to sophisticated error-prone US setups, this transduction principle suits for arbitrary pipe diameters and allows for an extremely simple design.

\section{Sensor Design}

Figure 1a depicts a schematic cross section of the sensor. It consists of a heater and two Pt100 elements, which measure the fluid $\left(T_{\mathrm{F}}\right)$ and heater temperature $\left(T_{\mathrm{H}}\right)$. Their difference (i.e., the heater excess temperature $\quad T=T_{\mathrm{H}}-T_{\mathrm{F}}$ ) correlates with the mean flow velocity $v$. For the sensor prototype, commercially available, miniaturized Pt100 elements were used (Figure 1b). The first Pt100 element measuring the heater temperature $\left(\mathrm{T}_{\mathrm{H}}\right)$ was placed on the copper pipe surface and fixed with cable ties. Subsequently, a thin copper wire was wound around it forming the heater, which was supplied by a constant electrical current (Figure 1c). The second Pt100 element was positioned upstream of the heater at a distance of $5 \mathrm{~cm}$ to acquire the fluid temperature $\left(T_{\mathrm{F}}\right)$. Around this temperature sensor, the same heater structure was built, however, without supplying it with electrical current. This "dummy heater" therefore does not influence a temperature field, but significantly reduces transient response after sudden variations of fluid temperature, since both temperature sensors feature approximately the same thermal mass. Finally, the whole pipe was insulated from the ambient.

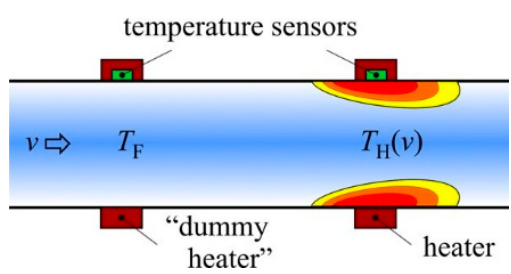

(a)

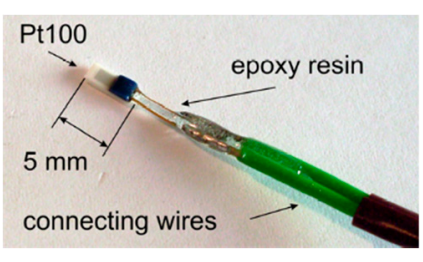

(b)

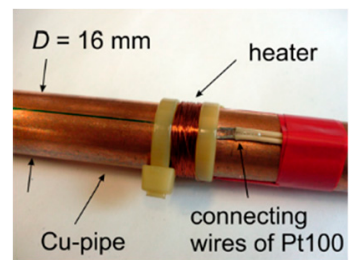

(c)

Figure 1. (a) Schematic cross section of the sensor; (b) Pt100 elements used for the sensor prototype; (c) A copper wire $(0.2 \mathrm{~mm}$ diameter, about $10 \mathrm{~m}$ long) was coiled around the downstream Pt100 element serving as a heater. The heater resistance amounts to approximately $11 \Omega$.

\section{FEM Simulations}

In order to analyze the flow conversion, a finite element method (FEM) model of the sensor was implemented (Figure 2a). The dimensions of the simulated $\mathrm{Cu}$-pipe are the same as for the prototype (16 mm diameter and $1 \mathrm{~mm}$ wall thickness). The sensor surface is considered to be ideally isolated from the ambient (adiabatic boundary condition). A constant heating power was induced at the heater and the temperature was recorded at the pipe surface below the heater $\left(T_{\mathrm{H}}\right)$ as well as $5 \mathrm{~cm}$ upstream of the heater $\left(T_{\mathrm{F}}\right)$. Figure $2 \mathrm{~b}$ shows the simulated heater excess temperature as a function of the mean flow velocity in the pipe as well as the actual measurement results obtained by means of the sensor prototype. 


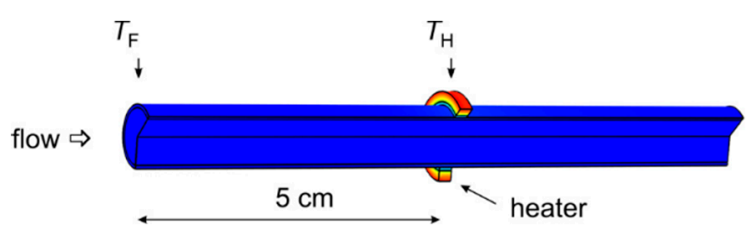

(a)

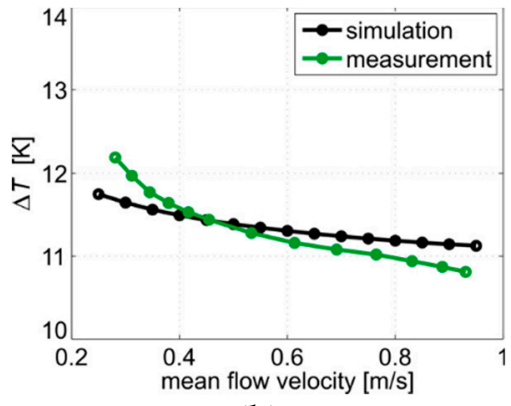

(b)

Figure 2. (a) FEM model of the sensor; (b) Heater excess temperature as a function of the mean flow velocity (simulation and measurement results at $7 \mathrm{~W}$ heating power).

\section{Sensor Electronics}

The dynamic of the excess temperature in Figure $2 b$ (i.e., the signal variation over the desired measurement range) lies in a range of only $1 \mathrm{~K}$ indicating that a high amplification and subsequent thorough signal conditioning must be applied. The signal conditioning circuit used for this purpose is depicted in Figure 3. It consists of a Wheatstone bridge and two amplifiers with optional offset correction. The electrical current through the Pt100 elements amounts to approximately $1 \mathrm{~mA}$ ensuring that self heating effects are minimized. When the offset correction of the first instrumentation amplifier is turned off, its output signal $U_{1}$ correlates to the heater excess temperature depicted in Figure $2 \mathrm{~b}$. For proper signal conditioning, the offset (denoted as offset1 in Figure 3) must be removed. The offset corrected signal is then normalized to the desired output range by the second amplifier in order to obtain the best readout and sensitivity.

Typically, the output signal UOUT depends on the fluid temperature $T_{\mathrm{F}}$. The exact temperature dependence is a function of many factors such as fluid parameters, pipe and heater material, or overall amplification $\left(\mathrm{A}_{1} \cdot \mathrm{A}_{2}\right)$ and must be determined experimentally. It can be accounted for, by measuring the fluid temperature and applying an appropriate offset at the second amplifier (denoted as offset $\left(T_{\mathrm{F}}\right)$ in Figure 3). Preliminary evaluation using the sensor prototype (water as a fluid, copper pipe and heater, $\mathrm{A}_{1}=1000, \mathrm{~A}_{2}=7.5$, offset $1 \approx 4.4 \mathrm{~V}$, heater supplied by a constant current, heating power approximately $7 \mathrm{~W}$ ) reveals a temperature coefficient in the range of a few percent per $1 \mathrm{~K}$ fluid temperature change. Thus, a signal correction must be applied in most applications.

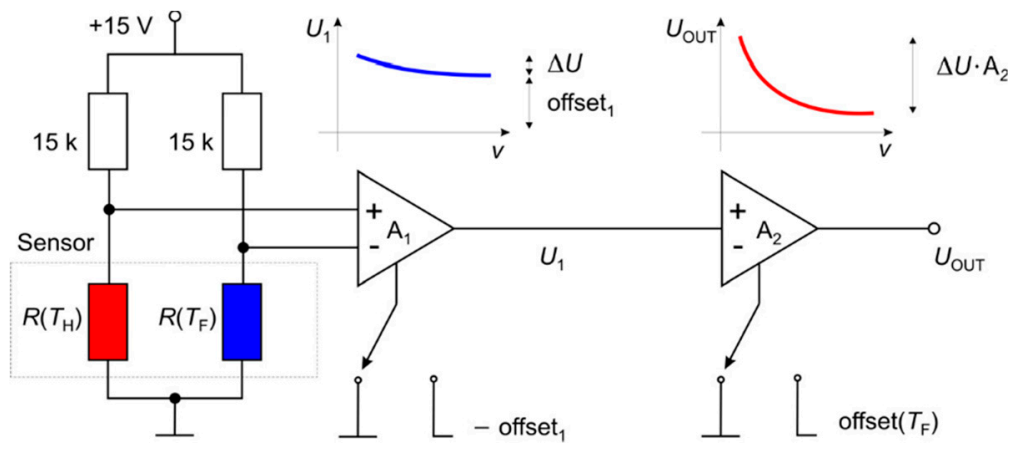

Figure 3. Schematic diagram of the signal conditioning circuit. $R\left(T_{\mathrm{H}}\right)$ and $R\left(T_{\mathrm{F}}\right)$ denote $\mathrm{Pt} 100$ elements located under the heater and upstream of the heater, respectively. Adjusting the offset of the second amplifier, the temperature dependence of the output signal can be taken into account.

\section{Results}

The sensor prototype was tested in a hydraulic system using water as test fluid. The deployed water pump allows for mean flow velocities in the sensor pipe up to $1 \mathrm{~m} / \mathrm{s}$. The overall amplification and the offset were chosen such that the sensor output signal fits between $0 \mathrm{~V}$ and $5 \mathrm{~V}$ in the flow range of interest (between $0.2 \mathrm{~m} / \mathrm{s}$ and $1 \mathrm{~m} / \mathrm{s}$ ). Hence, the output can easily be sampled with any commercially available microcontroller. 
The obtained conversion characteristic is depicted in Figure 4a. Applying the inverse function of the conversion characteristic from Figure $4 a$, the mean flow velocity inside the pipe can be calculated. The highest sensitivity is reached in the lower velocity range. For high flow velocities, the signal saturates and the sensitivity decreases.

In order to estimate the order of magnitude of the time constant, sudden changes of the volume flow were induced by fast adjusting (approx. $1 \mathrm{~s}$ ) of a pressure reduction valve. Figure $4 \mathrm{~b}$ shows a comparison of the sensor response for a typical clamp-on US sensor and the presented low-cost, non-invasive thermal flow sensor. Our prototype of the thermal flow sensor shows good accuracy but slower response time.

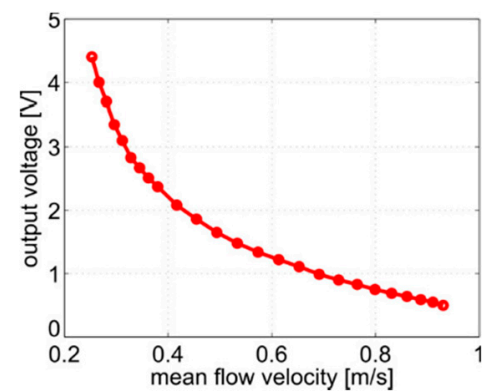

(a)

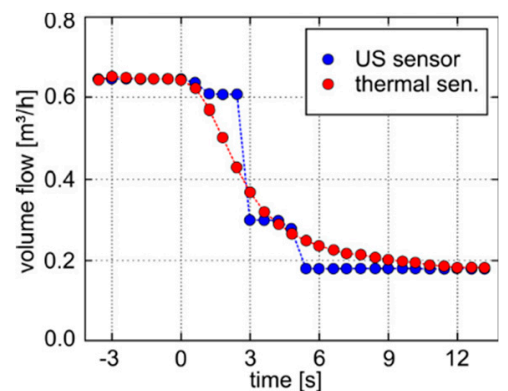

(b)

Figure 4. (a) Measured output characteristic of the non-invasive thermal flow sensor; (b) Comparison of the sensor response after a sudden change of the volume flow for a typical clamp-on US sensor and the non-invasive thermal flow sensor.

\section{Conclusions}

We presented a feasibility study on non-invasive flow rate measurements in metal pipes utilizing a thermal flow sensor. The proposed thermal flow transduction comprises two temperature sensors and a heater, all mounted on the outer pipe surface. The temperature difference of the Pt100 elements (i.e., the heater excess temperature) depends on the fluid velocity. However, a thorough signal conditioning must be applied to derive the mean volume flow inside the pipe.

The investigated sensor prototype consists of two miniaturized Pt100 elements with a copper wire wound around them. One coil was operated as a heater, whereas another one just serves as a "dummy" reducing the transient time. Practical realizations should comprise self adhesive Pt100 elements and heating stripes, which can be easily mounted on the pipe surface.

First measurement results demonstrate the feasibility of non-invasive measurements on metal pipes in hydraulic circuits by means of low-cost thermal flow transduction instead of high-priced clamp-on ultrasonic sensors. The sensor prototype was tested in a measurement range between 0.2 $\mathrm{m} / \mathrm{s}$ and $1 \mathrm{~m} / \mathrm{s}$. By appropriately adjusting the sensor electronics lower or higher flow range can also be achieved. The general drawback, however, is the saturation of the output characteristic with increasing flow velocity. Moreover, the output signal is temperature-dependent, so the variations of the fluid temperature during the measurements must be taken into account.

In comparison with a typical commercially available clamp-on US sensor, the presented noninvasive, low-cost thermal sensors features an extremely simple design and good accuracy, however, at the cost of a slower response time.

Acknowledgments: The presented work has been conducted in the course of the research project OptiMAS (FFG, research grant No. 854641), which is funded within the 3rd program "City of tomorrow" by the Austrian Ministry for Transport, Innovation and Technology. 


\section{References}

1. Perez-Lombard, L.; Ortiz, J.; Pout, C. A review on buildings energy consumption information. Energy Build. 2008, 40, 394-398.

2. Elwenspoek, M. Thermal flow micro sensors. In Proceedings of the CAS ‘99 1999 International Semiconductor Conference (Cat. No. 99TH8389), Sinaia, Romania, 5-9 October 1999; Volume 2, pp. 423-435.

3. Nguyen, N.T. Micromachined flow sensors-a review. Flow Meas. Instrum. 1997, 8, 7-16.

(C) 2018 by the authors. Licensee MDPI, Basel, Switzerland. This article is an open access article distributed under the terms and conditions of the Creative Commons Attribution (CC BY) license (http://creativecommons.org/licenses/by/4.0/). 